\title{
Synthesis and Antibacterial Activity of Urea and Thiourea Derivatives at C-8 Alkyl Chain of Anacardic Acid Mixture Isolated from a Natural Product Cashew Nut Shell Liquid (CNSL)
}

\author{
N. Subhakara Reddy ${ }^{1,2}$, A. Srinivas Rao ${ }^{1 *}$, M. Adharvana Chari ${ }^{3,4^{*}}$, V. Ravi Kumar ${ }^{1}$, V. Jyothi ${ }^{3}$, \\ V. Himabindu ${ }^{2}$ \\ ${ }^{1}$ Medicinal Chemistry Laboratory, GVK Biosciences Pvt. Ltd., Hyderabad, India \\ ${ }^{2}$ Centre for Environment, Institute of Science and Technology, JNT University, Hyderabad, India \\ ${ }^{3}$ Dr. MACS Bio-Pharma Pvt. Ltd., Hyderabad, India \\ ${ }^{4}$ Department of Complexity Science and Engineering, School of Frontier Sciences, \\ University of Tokyo, Chiba, Japan \\ E-mail:drmac_s@yahoo.com
}

Received August 12, 2011; revised September 23, 2011; accepted October 5, 2011

\begin{abstract}
Synthesis and antibacterial activity of some novel urea and thiourea derivatives (7a-7k, 8a-8f) of anacardic acid prepared from commercially available anacardic acid which is obtained from natural product Cashew Nut Shell Liquid (CNSL). Compounds (7a-7k, 8a-8f) were tested for Gram positive and Gram negative bacterial cultures. Most of the compounds were showed active compared with standard drug ampicilline.
\end{abstract}

Keywords: Synthesis, Urea and Thiourea Derivatives, Anacardic Acid, Anti-Bacterial Activity

\section{Introduction}

Among the different families of plants, Anacardiaceaeshrub family is very important since this plant consist of Non-isoprenoid phenolic lipids. The cashew tree, Anacardium occidentale $L$., is a botanical species native of eastern Brazil and was introduced into other tropical countries such as India, Africa, Indonesia and South East Asia in the 16th century [1-2]. Approximately $2-3 \mathrm{~cm}$ in length kidney shaped structure is true fruit of cashew is the nut, which is attached to the end of a fleshy bulb, generally called the cashew apple. The shell consist of the raw nut $(50 \%$ of the weight), the kernel $(25 \%)$ and the remaining $25 \%$ consists of the natural cashew nut shell liquid (CNSL), a viscous reddish brown liquid. The $\mathrm{CN}-$ $\mathrm{SL}$ is traditionally obtained as a by-product during the isolation of the kernel by roasting the raw nuts. Crude $\mathrm{CN}$ SL represents one of the major and cheapest sources of naturally occurring non-isoprenoid phenolic lipids such as anacardic acids (1), cardols (2), cardanols (3), methylcardols (4) (Figure 1) and polymeric materials. CNSL has found important commercial usage as the phenolic raw material for the manufacture of certain resins and plastics having unusual electric and frictional properties [3-6]. Anacardic acid mixture (1a-d) isolated from a natural product Cashew Nut Shell Liquid (CNSL) which is a by-product of cashew nut industry and these are salicylic acid derivatives with a nonisoprenoid alk(en)yl side chain [7]. Anacardic acid (pentadecyl salicylic acid) is a phenolic constituent present in Cashew Nut Shell Liquid (CNSL); (Anacardium occidentale L.) and exhibits antimicrobial properties [8-14], which have led to the preparation of various analogues [15-20] and soybean lipoxygenase-1 inhibitory activity [21-22] Kubo et al. [23] reported the separation of anacardic acid into monoene (15:1), diene $(15: 2)$ and triene $(15: 3)$ by preparative HPLC and tested against cancer cells, and found to show moderate cytotoxic activity on BT-20 breast and HeLa epithelioid cervix carcinoma cells. The emergence of drug resistant strains in clinical applications [24-26] especially to Gram positive bacteria[27-28] has created a problem of global proportions [29-30] G. C. Reddy et al. reported the synthesis of benzamide derivatives of anacardic acid [31], sildenafil analogues [32], dihydropyridine analogues [33] as calcium channel blockers, isonicotinoylhydrazones for antimycobacterial activity [34] starting from anacardic 


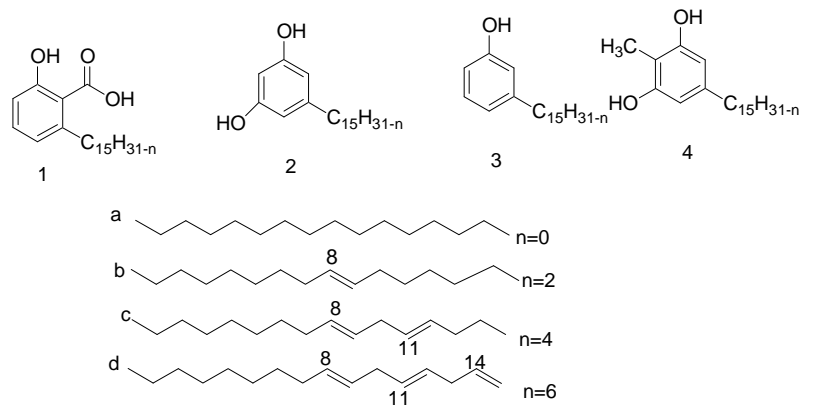

Figure 1. Naturally occurring non-isoprenoid phenolic lipids such as anacardic acids (1), cardols (2), cardanols (3) and methylcardols (4).

acid. Recently, a few anacardic acid derivatives exhibited various activities like affect the structure of the enzyme [35], anacardic acid is a specific activator of kinase activity of Aurora Kinase A [36], suppresses expression of nuclear factor-kB regulated gene products leading to potentiation of apoptosis [37] inhibitor of the HAT activeity of recombinant Plasmodium falciparum GCN5 [38] and as modulators of histone acetyltransferases [29]. Synthesis of lasiodiplodin from the non-isoprenoid phenolic lipids of CNSL as well as the salicylate macrolactone and other derivatives were reported by santos et al. [40-43].

In the present work we wish to report to synthesize novel cell permeable urea and thiourea compounds from cheaply available anacardic acid which was a major constituent of Cashew Nut Shell Liquid (CNSL) natural source to evaluate their biological activity by various anti- bacterial strains. This report describes the synthesis, spectroscopic identification and antibacterial activity of some novel urea and thiourea derivatives at C-8 alkyl chain of anacardic acids against Escherichia coli, Pseudomonas aeruginosa, Staphylococcus aureus and Streptococcus pyogenes bacterial strains.

\section{Results and Discussion}

Here we described the synthesis of various biologically active novel urea and thiourea derivatives using anacardic acid mixture as starting material and various reagents in the given below conditions (Scheme 1).

The anacardic acid mixture (1a-d) was isolated from commercially available CNSL by a reported method [44, 45]. Accordingly CNSL was treated with calcium hydroxide, during which anacardic acid present in CNSL becomes calcium anacardate, which was isolated and hydrolyzed with dil. hydrochloric acid to generate anacardic acid ene mixture, which was a mixture of monoene, diene and triene located at $\left(8^{\prime}\right),\left(8^{\prime}, 11^{\prime}\right)$ and $\left(8^{\prime}, 11^{\prime}, 14^{\prime}\right)$ of the $\mathrm{C} 15$ alkyl chain respectively. Anacardic acid ene mixture was methylated using dimethyl sulphate in presence of potassium carbonate in acetonitrile to afford 2. Ozonolysis of Compound 2 resulted in the formation of $\mathbf{3}, \mathrm{C} 8-\mathrm{OH}$. The compound $\mathbf{3}$ was converted to $\mathbf{4}$ using carbon tetra bromide in Dichloromethane. The compound 4 was reacted with sodium azide followed by reduction with $\mathrm{Pd} / \mathrm{C}$ under $\mathrm{H}_{2}$ pressure to obtaine amine 6 coupled with various isocynate or isothiocynate in chloroform to obtain compounds (7a-7k, 8a-8f, Scheme 1) of

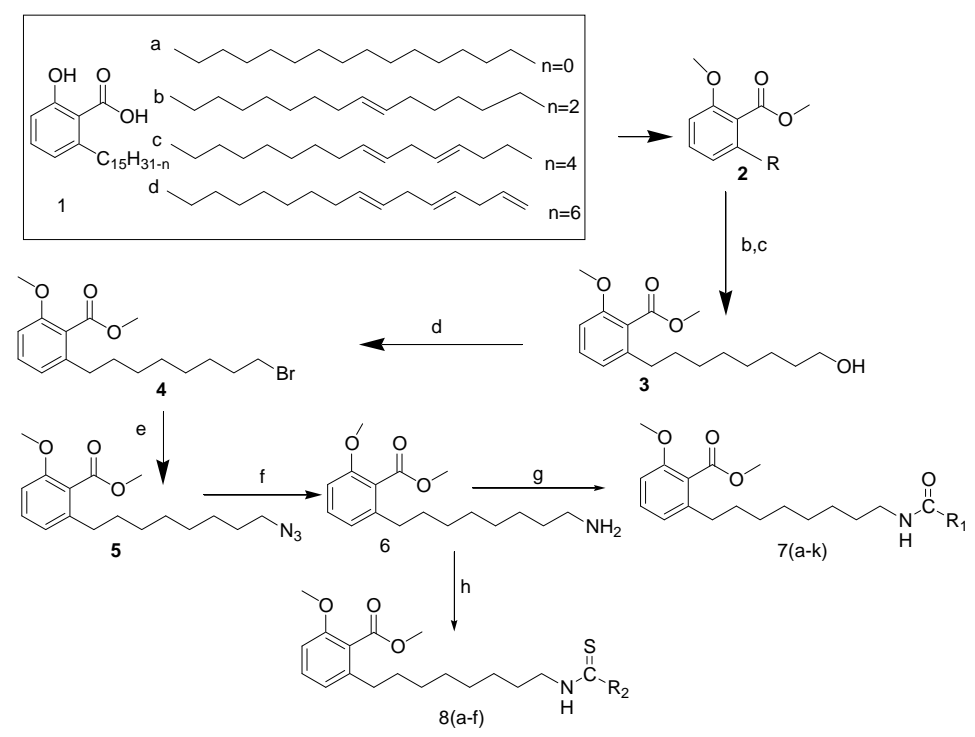

Scheme 1. Synthesis of various biologically active urea and thiourea (at $\mathrm{C8}$ alkyl chain) derivatives from anacardic acid mixture. Reagents: (a) Di methyl sulfate, $\mathrm{K}_{2} \mathrm{CO}_{3}$, Acetonitrile, $90^{\circ} \mathrm{C}, 24 \mathrm{~h}$; (b) Ozonalasis, $\mathrm{MeOH}, \mathrm{CH}_{2} \mathrm{Cl}_{2},-78^{\circ} \mathrm{C}, 6 \mathrm{~h}$; (c) $\mathrm{MeOH}$, $\mathrm{NaBH}_{4}, 18$ h, $0^{\circ} \mathrm{C}$, R.T; (d) $\mathrm{CBr}_{4}$, Pyridine, TPP, $\mathrm{CH}_{2} \mathrm{Cl}_{2}, 0^{\circ} \mathrm{C}, \mathrm{R} . \mathrm{T}, 8$ h; (e) $\mathrm{NaN}_{3}, \mathrm{DMF}, 100^{\circ} \mathrm{C}, 4$ h; (f) $10 \% \mathrm{Pd} / \mathrm{C}, 50$ psi, 2 h; (g) different isocyanate, $\mathrm{CHCl}_{3}$; (h) different isothiocyanate, $\mathrm{CHCl}_{3}$. 
urea and thiourea derivatives were purified by column chromatography to yield title compounds. The structure of urea and thiourea derivatives (7a-7k, 8a-8f) was determined by using different spectroscopic techniques ${ }^{1} \mathrm{H}$ NMR, IR, Mass. The resulting compounds are screened for their antibacterial activity.

\section{Biological Activity}

The urea and thiourea derivatives (7a-7k, 8a-8f) were screened for their antibacterial activity [27] against some of the pathogenic bacteria viz. E. coli (MTCC443), P. aeruginosa (MTCC424), S. aureus, (MTCC96) and S. pyogenes (MTCC443) using agar well diffusion method according to the literature protocol [46]. The anti-bacterial activity of the analogues was compared with standard drug ampicilline and the results of investigation have been presented in Table $\mathbf{1}$ and observed that some of the compounds are showed high biological activity.

Table 1. Antibacterial activity of urea and thiourea derivatives at C-8 alkyl chain of anacardic acid mixture.

\begin{tabular}{|c|c|c|c|c|c|}
\hline \multirow{2}{*}{ Compound No. } & \multirow{2}{*}{$\mathrm{R}$} & \multicolumn{4}{|c|}{ Name of the Bacteria (Conc. $250 \mu \mathrm{g} / \mathrm{ml}$ ) \& Inhibition Zone in $\mathrm{mm}$} \\
\hline & & E. coli MTCC443 & P. aeruginosa MTCC424 & S. aureus MTCC96 & S.pygenes MTCC442 \\
\hline S* ampicilline & SD* amplicilline & 20 & 20 & 18 & 19 \\
\hline $7 \mathrm{a}$ & & 17 & 16 & 17 & 16 \\
\hline $7 \mathrm{~b}$ & & 19 & 19 & 15 & 19 \\
\hline $7 \mathrm{c}$ & & 21 & 18 & 17 & 17 \\
\hline $7 d$ & & 19 & 16 & 18 & 15 \\
\hline $7 \mathrm{e}$ & & 17 & 16 & 18 & 19 \\
\hline $7 f$ & & 19 & 18 & 17 & 15 \\
\hline $7 \mathrm{~g}$ & & 20 & 17 & 17 & 17 \\
\hline $7 \mathrm{~h}$ & & 17 & 17 & 17 & 19 \\
\hline $7 \mathrm{i}$ & & 17 & 16 & 16 & 17 \\
\hline $7 \mathrm{j}$ & & 18 & 17 & 17 & 15 \\
\hline $7 \mathrm{k}$ & & 16 & 16 & 16 & 16 \\
\hline $8 \mathrm{a}$ & & 16 & 21 & 17 & 17 \\
\hline $8 b$ & & 19 & 17 & 17 & 18 \\
\hline $8 \mathrm{c}$ & & 17 & 16 & 16 & 18 \\
\hline $8 \mathrm{~d}$ & & 16 & 20 & 15 & 17 \\
\hline $8 \mathrm{e}$ & & 22 & 20 & 16 & 17 \\
\hline $8 \mathrm{f}$ & & 15 & 16 & 17 & 19 \\
\hline
\end{tabular}


Based on the test results it is evident that several of synthesized anacardic acid analogues possess moderate to good activity against the Gram +ve and Gram -ve bacteria. Of all the compounds prepared entities $\mathbf{7 a}, \mathbf{7 b}, \mathbf{7 c}, \mathbf{7 d}$, $\mathbf{7 f}, \mathbf{7 g}, \mathbf{8 b}$ and $\mathbf{8 e}$ activity against $E$. coli, MTCC443, 7a, $\mathbf{7 b}, \mathbf{8 a}, \mathbf{8 d}$ and $\mathbf{8 e}$ activity against $P$. aeruginosa, MTCC424; display good to excellent activity while the remaining compounds showed moderate activity. The most active antibacterial agent against Escherichia coli found to be compound $\mathbf{7 c}, \mathbf{7 f}, \mathbf{7 g}$, and $\mathbf{8 e}$ having $-C N, F$ groups and other compounds in the series exhibited moderate to good activity. The compounds $\mathbf{7 d}, \mathbf{7 e}, \mathbf{8 b}$ and $\mathbf{8 f}$ showed good activity against $S$. aureus MTCC96 and 7a, 7b, 7e, $\mathbf{7 h}, \mathbf{8 b}, \mathbf{8 c}$ and $\mathbf{8 f}$ showed good activity against $S$. pyogenes MTCC442. This indicates chloro, dichloro, flouro, cyano and methoxy substituted compounds showed better activity when compared to other substituted groups. Here seems to be, thiourea substituted novel compounds are exhibiting better activity than urea substituted compounds. The activity depends to some extent on the $\mathrm{R}$ substituent, however all the compounds showed antibacterial activity. It may be suggested that the anacardic acid derivative with a suitable $\mathrm{R}$ may lead to a good antibacterial agent against all the Escherichia coli, Pseudomonas aeruginosa, Staphylococcus aureus and Streptococcus pyogenes bacterial strains.

\section{Conclusions}

In summary, the present study describes a convenient and efficient protocol for the synthesis of sulfonamide derivatives by using anacardic acid mixture using various reagents and different conditions. We believe that this procedure is convenient, economic and a user-friendly process for the synthesis of these various novel urea and thiourea compounds from anacardic acid mixture. All compounds structures are supported by physico chemical and IR, NMR, Mass spectral data. Urea and thiourea derivatives were screened for their antibacterial activity against few bacterial strains and observed that some of the compounds are showed more biological activity than standards used.

\section{Experimental Section}

\subsection{General Reagents and Equipment}

All chemicals and solvents were obtained from Aldrich and Spectrochem., India and used without further purification. Column chromatographic separations were carried out on silica gel 60 - 120 mesh size and eluting with a gradient of hexane: ethyl acetate. Analytical thin layer chromatography was performed on precoated Merck si- lica gel (60F254/0.2 mm) plates using UV light, 5\% ethanolic phosphomolybdic acid or iodine vapours to visualize the spots. Melting points were determined in open glass capillaries on a Mel-temp apparatus and are uncorrected. The IR spectra were recorded on a Thermo Nicolet IR 200 FT-IR spectrometer as $\mathrm{KBr}$ pellets and the wave numbers were given in $\mathrm{cm}^{-1}$. The ${ }^{1} \mathrm{H}$ and ${ }^{13} \mathrm{C}$ NMR spectra of samples were recorded on a Varian EM-360, NMR spectrometer using TMS as an internal standard in $\mathrm{CDCl}_{3}$. The mass spectra were recorded on Jeol JMS-D 300 and Finnigan Mat $b$ at $70 \mathrm{eV}$ with an emission current of $100 \mu \mathrm{A}$. The oxidative cleavages were performed with a Welsbach T-408 ozonizator and the catalytic hydrogenations in a Parr apparatus.

\subsection{General Procedure: Isolation of Anacardic Acids (1)}

The shells $(500 \mathrm{~g})$ of cashew nuts from Anacardiumoccidentale were extracted in a Soxhletextractor with commercial $95 \%$ ethanol $(2.0 \mathrm{~L})$ during $6 \mathrm{~h}$, yielding a crude extract (CNSL, $157 \mathrm{~g}, 31 \%$ by weight). Anacardic acids (1) were removed in $61 \%$ from CNSL (15.25 g) either by precipitation with lead nitrate or calcium hydroxide according to protocols described in the literature $[44,45]$. The spectral properties were identical to those reported in the literature $[44,45]$.

Preparation of Methyl anacardate methyl ethers ene mixture (2): To a solution of Compound 1 (65 g, $186.78 \mathrm{mmol})$ in acetone was added $\mathrm{K}_{2} \mathrm{CO}_{3}(103.1 \mathrm{~g}$, $747.12 \mathrm{mmol})$, Di methyl sulfate $(44.3 \mathrm{~mL}, 466.95$ mmol). The contents were heated at $65^{\circ} \mathrm{C}$ for $5 \mathrm{~h}$. Reaction mixture was cooled to room temperature, filtered and washed with ethyl acetate. Filtrate was distilled off, crude compound was re dissolved in ethyl acetate (300 $\mathrm{mL}$ ). Organic layer was washed with water, brine solution and dried over anhydrous sodium sulphate and distilled off ethyl acetate. Crude compound was purified by 60 - 120 silica pet ether pack column compound was eluted with $5 \%$ ethyl acetate: pet ether to get compound 2, Yield: $58 \mathrm{~g}$, light yellow liquid.

Synthesis of 2-(8-Hydroxy-octyl)-6-methoxybenzoic acid methyl ester (3): A solution of compound 2 (15 $\mathrm{g}, 40.540 \mathrm{mmol})$ in dichloro methane: methanol $(1: 1,500$ $\mathrm{mL}$ ) was added a pinch of Sudan red catalyst and cooled to $-78^{\circ} \mathrm{C}$. Ozone gas purged through reaction mixture until starting material was completed $(8 \mathrm{~h})$. Nitrogen gas was purged through reaction mixture for $30 \mathrm{~min}$ (to remove excess $\mathrm{O}_{3}$ gas), dimethyl sulfide was added few drops and stirred for $20 \mathrm{~min}$ at $-15^{\circ} \mathrm{C}$. Sodium borohydride (9.970 g, $263.51 \mathrm{mmol})$ was added portion wise over a period of $45 \mathrm{~min}$. Reaction mixture was slowly bring it to room temperature and stirred at this tempera- 
ture for $18 \mathrm{~h}$. Reaction mixture was quenched with cold water $(400 \mathrm{~mL})$, dichloro methane and methanol distilled off and crude compound was diluted with water and extracted with ethyl acetate $(2 \times 200 \mathrm{~mL})$. The combined organic layer was washed with brine solution $(150 \mathrm{~mL})$ dried over anhydrous sodium sulphate, filtered and evaporated under vacuum, to obtaine crude compound was purified by neutral alumina pet ether packed column, compound was eluted with $20 \%$ ethyl acetate: pet ether to obtain compound (3) as yellow liquid (7.1 g, 59.5\%); IR (DCM film): 3401, 2930, 1728, 1586, 1467, 1268, 1110, 1071, 954, $749 \mathrm{~cm}^{-1} ;{ }^{1} \mathrm{H} \mathrm{NMR}\left(\mathrm{CDCl}_{3}, 400 \mathrm{MHz}\right)$ : $\delta 1.31(\mathrm{bs}, 8 \mathrm{H}), 1.53-1.59(\mathrm{~m}, 4 \mathrm{H}), 2.53(\mathrm{t}, 2 \mathrm{H}, \mathrm{J}=8.0$ $\mathrm{Hz}), 3.63(\mathrm{t}, 2 \mathrm{H}, \mathrm{J}=6.8 \mathrm{~Hz}), 3.81(\mathrm{~s}, 3 \mathrm{H}), 3.90(\mathrm{~s}, 3 \mathrm{H})$, $6.75(\mathrm{~d}, 1 \mathrm{H}, \mathrm{J}=8.4 \mathrm{~Hz}), 6.82(\mathrm{~d}, 1 \mathrm{H}, \mathrm{J}=7.6 \mathrm{~Hz}), 7.26$ $7.28(\mathrm{~m}, 1 \mathrm{H}) \mathrm{ppm} ;{ }^{13} \mathrm{C} \mathrm{NMR}\left(100 \mathrm{MHz}, \mathrm{CDCl}_{3}\right), \delta$ : 25.58, 29.16, 29.23, 29.63, 31.00, 32.62, 33.35, 52.10, 55.73, 62.81, 108.23, 121.37, 123.26, 130.18, 141.17, 156.10, 168.93 ppm; ESIMS (m/z): $295(\mathrm{M}+\mathrm{H})^{+}$.

Synthesis of 2-(8-bromo-octyl)-6-methoxy-benzoic acid methyl ester (4): A solution of compound 2 (15 g, $51.02 \mathrm{mmol})$ in Dichloro methane $(150 \mathrm{~mL})$ was added dry pyridine $(42 \mathrm{~mL}, 510.2 \mathrm{mmol})$ tri phenyl phosphene $(22.73 \mathrm{~g}, 86.734 \mathrm{mmol})$ at $0^{\circ} \mathrm{C}$. Carbon tetra bromide $(25.4 \mathrm{~g}, 76.53 \mathrm{mmol})$ was added portion wise over a period of $15 \mathrm{~min}$. The contain were slowly bring it to $\mathrm{rt}$ and stirred at $\mathrm{rt}$ for $6 \mathrm{~h}$. Reaction mixture was diluted with DCM $(100 \mathrm{~mL})$ washed with $2 \mathrm{~N} \mathrm{HCl}(2 \times 150 \mathrm{~mL})$, water $(200 \mathrm{~mL})$, brine solution $(175 \mathrm{~mL})$, dried over anhydrous $\mathrm{Na}_{2} \mathrm{SO}_{4}$, filtered and evaporated under vacuum, Crude compound was purified by 100 - 200 silica pet ether column compound was eluted with $10 \%$ ethyl acetate: pet ether and distilled off solvent to obtain compound $\mathbf{4}$ (16.5 g, 90.8\%) as yellow liquid; IR (DCM film): 3071, 3002, 2931, 2854, 1732, 1588, 1464, 1437, 1268, 1109, 1072, 960, $749 \mathrm{~cm}^{-1}$; ${ }^{1} \mathrm{H}$ NMR $\left(\mathrm{CDCl}_{3}, 400 \mathrm{MHz}\right): \delta$ $1.29-1.42(\mathrm{~m}, 8 \mathrm{H}), 1.52-1.59(\mathrm{~m}, 4 \mathrm{H}), 1.80-1.87(\mathrm{~m}$, $2 \mathrm{H}), 2.53(\mathrm{t}, 2 \mathrm{H}, \mathrm{J}=8.0 \mathrm{~Hz}), 3.40(\mathrm{t}, 2 \mathrm{H}, \mathrm{J}=7.2 \mathrm{~Hz})$, $3.82(\mathrm{~s}, 3 \mathrm{H}), 3.90(\mathrm{~s}, 3 \mathrm{H}), 6.76(\mathrm{~d}, 1 \mathrm{H}, \mathrm{J}=8.4 \mathrm{~Hz}), 6.81$ $(\mathrm{d}, 1 \mathrm{H}, \mathrm{J}=7.6 \mathrm{~Hz}), 7.24-7.28(\mathrm{~m}, 1 \mathrm{H}) \mathrm{ppm} ;{ }^{13} \mathrm{C} \mathrm{NMR}$ (100 MHz, $\mathrm{CDCl}_{3}$ ), $\delta: 28.05,28.56,29.12,29.26,31.02$, $32.72,33.36,34.02,52.13,55.78,108.27,121.39,123.32$, 130.20, 141.14, 156.14, 168.90 ppm; $\operatorname{ESIMS(m/z):~} 357$ $(\mathrm{M}+\mathrm{H})^{+}, 359$ (bromo).

Synthesis of 2-(8-aza-octyl)-6-methoxy-benzoic acid methyl ester (5): A solution of compound 3 (2.0 g, $5.617 \mathrm{mmol})$ in DMF $(10 \mathrm{~mL})$ was added Sodium azide (548 mg, 8.426). The contain were heated at $100^{\circ} \mathrm{C}$ for $3 \mathrm{~h}$, reaction mixture was poured into cool water $(70 \mathrm{~mL})$ and extracted with diethyl ether $(2 \times 40 \mathrm{~mL})$, the organic layer was washed with water $(50 \mathrm{~mL})$, brine solution $(30$ $\mathrm{mL}$ ), dried over anhydrous $\mathrm{Na}_{2} \mathrm{SO}_{4}$, filtered and evaporated under vacuum to obtain compound 5 as yellow liq- uid (1.5 g, 83.7\%); IR (DCM film): 3087, 3002, 2932, 2856, 2095, 1731, 1588, 1464, 1266, 1109, 1071, 753 $\mathrm{cm}^{-1} ;{ }^{1} \mathrm{H}$ NMR $\left(\mathrm{CDCl}_{3}, 400 \mathrm{MHz}\right): \delta 1.30(\mathrm{bs}, 8 \mathrm{H}), 1.59$ (bs, 4H), 2.53 (t, 2H, J = 7.6 Hz), 3.25 (t, 2H, J=7.2 Hz), $3.82(\mathrm{~s}, 3 \mathrm{H}), 3.91(\mathrm{~s}, 3 \mathrm{H}), 6.76(\mathrm{~d}, 1 \mathrm{H}, \mathrm{J}=8.4 \mathrm{~Hz}), 6.82$ $(\mathrm{d}, 1 \mathrm{H}, \mathrm{J}=8.0 \mathrm{~Hz}), 7.25$ - $7.29(\mathrm{~m}, 1 \mathrm{H})$ ppm; ESIMS $(\mathrm{m} / \mathrm{z}): 320(\mathrm{M}+\mathrm{H})^{+}$.

Synthesis of 2-(8-Amino-octyl)-6-methoxy-benzoic acid methyl ester (6): A solution of compound 4 (2.0 g, $\mathrm{mmol})$ in ethanol $(30 \mathrm{~mL})$ was taken into a $500 \mathrm{~mL}$ Parrhydrogenation vessel and added a suspension of $10 \% \mathrm{Pd} / \mathrm{C}$ $(220 \mathrm{mg}, 10 \%)$ in $20 \mathrm{~mL}$ of ethanol under argon atmosphere and applied $\mathrm{H}_{2}$-pressure (60 psi) for $2 \mathrm{~h}$. Reaction mixture was filtered through celite bed and concentrated the filtrate under reduced pressure to obtain 2-(8-Amino-octyl)-6-methoxy-benzoic acid methyl ester (6) (1.7 g, $92.5 \%$ ) as a yellow liquid. IR (neat): 3436, 2931, 2857, $1729,1587,1467,1438,1268,1111,1073,829,753$ $\mathrm{cm}^{-1}$; ${ }^{1} \mathrm{H}$ NMR (CDCl3, $\left.400 \mathrm{MHz}\right): \delta 1.273(\mathrm{bs}, 8 \mathrm{H})$, 1.54 (bs, 2H), $1.65-1.79(\mathrm{~m}, 2 \mathrm{H}), 2.51(\mathrm{t}, 2 \mathrm{H}, \mathrm{J}=7.6$ $\mathrm{Hz}), 2.94$ (t, 2H, J = 8.0 Hz), 3.79 (s, 3H), 3.89 (s, 3H), $6.74(\mathrm{~d}, 1 \mathrm{H}, \mathrm{J}=8.0 \mathrm{~Hz}), 6.80(\mathrm{~d}, 1 \mathrm{H}, \mathrm{J}=8.0 \mathrm{~Hz}), 7.23-$ $7.27(\mathrm{~m}, 1 \mathrm{H}) \mathrm{ppm}$; ESIMS (m/z): $294(\mathrm{M}+\mathrm{H})^{+}$.

Synthesis of urea and thio urea compounds: A solution of amine $(300 \mathrm{mg}, 1.023 \mathrm{mmol})$ in dry $\mathrm{CHCl}_{3}$ was taken in seal tube was added isocynate or iso thiocynate $(1.22 \mathrm{mmol})$ at $\mathrm{rt}$ and stirred at $\mathrm{rt}$ for $3 \mathrm{~h}$ to $8 \mathrm{~h}$ and distilled off solvent and crude compound was purified by column.

Synthesis of methyl 2-(8-(3-ethylureido)octyl)-6-methoxybenzoate (7a): Using 6 and ethyl isocyanate as starting materials, the title compound 7a was obtained as a off white solid (Yield $=44.4 \%$ ); m.p. $71^{\circ} \mathrm{C}-72^{\circ} \mathrm{C}$; IR (KBr): 3338, 2929, 2854, 1729, 1625, 1579, 1466, 1269, 1108, 1072, $738 \mathrm{~cm}^{-1} ;{ }^{1} \mathrm{H}$ NMR $\left(\mathrm{CDCl}_{3}, 400 \mathrm{MHz}\right): \delta$ $1.11-1.15(\mathrm{~m}, 3 \mathrm{H}), 1.28$ (bs, 8H), $1.45-1.59$ (m, 4H), $2.53(\mathrm{t}, 2 \mathrm{H}, \mathrm{J}=8.0 \mathrm{~Hz}), 3.12-3.24(\mathrm{~m}, 4 \mathrm{H}), 3.82(\mathrm{~s}, 3 \mathrm{H})$, $3.91(\mathrm{~s}, 3 \mathrm{H}), 4.23(\mathrm{~s}, 2 \mathrm{H}), 6.76(\mathrm{~d}, 1 \mathrm{H}, \mathrm{J}=8.4 \mathrm{~Hz}), 6.81$ $(\mathrm{d}, 1 \mathrm{H}, \mathrm{J}=7.2 \mathrm{~Hz}), 7.25-7.29(\mathrm{~m}, 1 \mathrm{H}) \mathrm{ppm} ;{ }^{13} \mathrm{C} \mathrm{NMR}$ $\left(100 \mathrm{MHz}, \mathrm{CDCl}_{3}\right)$, $\delta: 15.46,26.73,29.07,29.18,29.21$, $30.14,30.94,33.35,35.01,40.25,52.10,55.78,108.33$, $121.43,123.30,130.24,141.17,156.15,158.65,169.05$ ppm; ESIMS (m/z): $365(\mathrm{M}+\mathrm{H})^{+}$.

Synthesis of methyl 2-(8-(3-cyclopentylureido)octyl) -6-methoxybenzoate (7b): Using 6 and cyclopentyl isocyanate as starting materials, the title compound $\mathbf{7 b}$ was obtained as a white solid (Yield $=60.4 \%$ ); m.p. $80^{\circ} \mathrm{C}$ $81^{\circ} \mathrm{C}$; IR (DCM film): 3339, 2932, 2858, 1731, 1630, 1574, 1466, 1267, 1110, 1073, $749 \mathrm{~cm}^{-1}$; ${ }^{1} \mathrm{H}$ NMR (CD$\left.\mathrm{Cl}_{3}, 400 \mathrm{MHz}\right): \delta 1.28-1.65(\mathrm{~m}, 18 \mathrm{H}), 1.92-2.00(\mathrm{~m}$, 2H), 2.53 (t, 2H, J = 7.6 Hz), $3.12-3.16$ (q, 2H), $3.81(\mathrm{~s}$, $3 \mathrm{H}), 3.90(\mathrm{~s}, 3 \mathrm{H}), 3.91-3.95(\mathrm{~m}, 1 \mathrm{H}), 4.31(\mathrm{~s}, 1 \mathrm{H}), 6.75$ $(\mathrm{d}, 1 \mathrm{H}, \mathrm{J}=8.4 \mathrm{~Hz}), 6.81(\mathrm{~d}, 1 \mathrm{H}, \mathrm{J}=8.0 \mathrm{~Hz}), 7.25-7.29$ 
(m, 1H) ppm; ${ }^{13} \mathrm{C}$ NMR (100 MHz, $\left.\mathrm{CDCl}_{3}\right), \delta: 23.51$, 26.78, 29.09, 29.19, 29.23, 30.21, 30.96, 33.35, 33.51, $40.24,51.86,52.09,55.77,108.31,121.42,123.30$, $130.23,141.16,156.15,158.34,169.01$ ppm; ESIMS $(\mathrm{m} / \mathrm{z}): 405(\mathrm{M}+\mathrm{H})^{+}$.

Synthesis of methyl 2-methoxy-6-(8-(3-phenylureido)octyl) benzoate (7c): Using 6 and phenyl isocyanate as starting materials, the title compound 7c was obtained as a cream color semi solid (Yield $=66.2 \%$ ); IR (DCM film): 3345, 3298, 3085, 3008, 2929, 2853, 1729, $1637,1589,1552,1467,1270,1111,1071,735 \mathrm{~cm}^{-1} ;{ }^{1} \mathrm{H}$ NMR $\left(\mathrm{CDCl}_{3}, 400 \mathrm{MHz}\right): \delta 1.26(\mathrm{bs}, 8 \mathrm{H}), 1.43-1.55(\mathrm{~m}$, $4 \mathrm{H}), 2.53(\mathrm{t}, 2 \mathrm{H}, \mathrm{J}=8.0 \mathrm{~Hz}), 3.17-3.22(\mathrm{q}, 2 \mathrm{H}), 3.80(\mathrm{~s}$, $3 \mathrm{H}), 3.91$ (s, 3H), 5.07 (bs, 1H), $6.76(\mathrm{~d}, 1 \mathrm{H}, \mathrm{J}=8.4 \mathrm{~Hz})$, $6.81(\mathrm{~d}, 1 \mathrm{H}, \mathrm{J}=8.0 \mathrm{~Hz}), 7.01-7.05(\mathrm{~m}, 1 \mathrm{H}), 7.24-7.31$ $(\mathrm{m}, 5 \mathrm{H}) \mathrm{ppm} ; \operatorname{ESIMS}(\mathrm{m} / \mathrm{z}): 413(\mathrm{M}+\mathrm{H})^{+}$.

Synthesis of methyl 2-(8-(3-(3-chlorophenyl)ureido) octyl)-6-methoxybenzoate (7d): Using 6 and 3-chlorophenyl isocyanate as starting materials, the title compound 7d was obtained as a white color solid (Yield = $35 \%$ ); m.p. $102^{\circ} \mathrm{C}-103^{\circ} \mathrm{C}$; IR (DCM film): 3347, 3080, 3004, 2930, 2855, 1729, 1659, 1590, 1549, 1473, 1429, 1268, 1110, 1073, $766 \mathrm{~cm}^{-1} ;{ }^{1} \mathrm{H}$ NMR $\left(\mathrm{CDCl}_{3}, 400\right.$ MHz): $\delta 1.27(\mathrm{bs}, 8 \mathrm{H}), 1.40-1.60(\mathrm{~m}, 4 \mathrm{H}), 2.54(\mathrm{t}, 2 \mathrm{H}, \mathrm{J}$ $=7.6 \mathrm{~Hz}), 3.19-3.24(\mathrm{q}, 2 \mathrm{H}), 3.80(\mathrm{~s}, 3 \mathrm{H}), 3.92(\mathrm{~s}, 3 \mathrm{H})$, $5.04(\mathrm{~s}, 1 \mathrm{H}), 6.76-6.85(\mathrm{~m}, 3 \mathrm{H}), 6.97(\mathrm{~d}, 1 \mathrm{H}, 7.2 \mathrm{~Hz})$, 7.14 - $7.37(\mathrm{~m}, 4 \mathrm{H}) \mathrm{ppm}$; ESIMS (m/z): $447(\mathrm{M}+\mathrm{H})^{+}$.

Synthesis of methyl 2-(8-(3-(3,4-dichlorophenyl)ureido)octyl)-6-methoxybenzoate (7e): Using 6 and 3, 4 dichlorophenyl isocyanate as starting materials, the title compound 7e was obtained as a light brown solid (Yield $=65 \%$ ); m.p. $80^{\circ} \mathrm{C}-82^{\circ} \mathrm{C}$; IR (DCM film): 3351, 3099, 2930, 2855, 1729, 1661, 1587, 1542, 1470, 1381, 1270, $1115,1072,1028,820,746 \mathrm{~cm}^{-1} ;{ }^{1} \mathrm{H}$ NMR $\left(\mathrm{CDCl}_{3}, 400\right.$ $\mathrm{MHz}): \delta 1.25$ (bs, 8H), $1.41-1.58(\mathrm{~m}, 4 \mathrm{H}), 2.55(\mathrm{t}, 2 \mathrm{H}, \mathrm{J}$ $=8.0 \mathrm{~Hz}), 3.17-3.22(\mathrm{q}, 2 \mathrm{H}), 3.80(\mathrm{~s}, 3 \mathrm{H}), 3.93(\mathrm{~s}, 3 \mathrm{H})$, $5.15(\mathrm{~s}, 1 \mathrm{H}), 6.77(\mathrm{~d}, 1 \mathrm{H}, \mathrm{J}=8.0 \mathrm{~Hz}), 6.82(\mathrm{~d}, 1 \mathrm{H}, \mathrm{J}=$ $7.6 \mathrm{~Hz}), 7.08$ - 7.19 (m, 2H), $7.20-7.31$ (m, 2H), 7.48 (s, 1H) ppm; ESIMS (m/z): $481(\mathrm{M}+\mathrm{H})^{+} .483$ (chloro).

Synthesis of methyl 2-(8-(3-(2-fluorophenyl)ureido) octyl)-6-methoxybenzoate (7f): Using 6 and 2-fluorophenyl isocyanate as starting materials, the title compound 7f was obtained as a light brown solid (Yield = 68.2\%); m.p. $92^{\circ} \mathrm{C}-93^{\circ} \mathrm{C}$; IR (DCM film): 3348, 3073, 3006, 2930, 2855, 1730, 1658, 1551, 1457, 1265, 1188, 1109, 1073, $810 \mathrm{~cm}^{-1} ;{ }^{1} \mathrm{H}$ NMR $\left(\mathrm{CDCl}_{3}, 400 \mathrm{MHz}\right): \delta$ $1.28(\mathrm{bs}, 8 \mathrm{H}), 1.42-1.60(\mathrm{~m}, 4 \mathrm{H}), 2.54(\mathrm{t}, 2 \mathrm{H}, \mathrm{J}=7.6$ $\mathrm{Hz}), 3.21-3.25$ (q, 2H), 3.80 (s, 3H), 3.91 (s, 3H), 5.04 $(\mathrm{s}, 1 \mathrm{H}), 6.67(\mathrm{~s}, 1 \mathrm{H}), 6.75(\mathrm{~d}, 1 \mathrm{H}, \mathrm{J}=8.0 \mathrm{~Hz}), 6.81(\mathrm{~d}$, $1 \mathrm{H}, \mathrm{J}=8.0 \mathrm{~Hz}), 6.92-6.98(\mathrm{~m}, 1 \mathrm{H}), 7.01-7.10(\mathrm{~m}, 2 \mathrm{H})$, $7.24-7.29(\mathrm{~m}, 1 \mathrm{H}), 8.01-8.10(\mathrm{~m}, 1 \mathrm{H}) \mathrm{ppm}$; ESIMS $(\mathrm{m} / \mathrm{z}): 431(\mathrm{M}+\mathrm{H})^{+}$.

Synthesis of methyl 2-(8-(3-(2-cyanophenyl)ureido) octyl)-6-methoxybenzoate (7g): Using 6 and 2-cyanophenyl isocyanate as starting materials, the title compound $\mathbf{7 g}$ was obtained as a light green semi solid (Yield =26.7\%); IR (DCM film): 3346, 3078, 3000, 2930, 2855, 2221, 1728, 1662, 1581, 1546, 1452, 1268, 1110, 1072, $758 \mathrm{~cm}^{-1}$; ${ }^{1} \mathrm{H} \mathrm{NMR}\left(\mathrm{CDCl}_{3}, 400 \mathrm{MHz}\right): \delta 1.30$ (bs, 8H), $1.51-1.57(\mathrm{~m}, 4 \mathrm{H}), 2.54(\mathrm{t}, 2 \mathrm{H}, \mathrm{J}=7.6 \mathrm{~Hz}), 3.23-3.28$ $(\mathrm{q}, 2 \mathrm{H}), 3.80(\mathrm{~s}, 3 \mathrm{H}), 3.91(\mathrm{~s}, 3 \mathrm{H}), 5.33(\mathrm{~s}, 1 \mathrm{H}), 6.75$ (d, $1 \mathrm{H}, \mathrm{J}=8 \mathrm{~Hz}), 6.81(\mathrm{~d}, 1 \mathrm{H}, \mathrm{J}=7.6 \mathrm{~Hz}), 7.02(\mathrm{t}, 1 \mathrm{H}, \mathrm{J}=$ $7.6 \mathrm{~Hz}), 7.11(\mathrm{~s}, 1 \mathrm{H}), 7.24-7.28(\mathrm{~m}, 1 \mathrm{H}), 7.49-7.54(\mathrm{~m}$, 2H), $8.31(\mathrm{~d}, 1 \mathrm{H}, \mathrm{J}=8.0 \mathrm{~Hz})$ ppm; ESIMS (m/z): $436(\mathrm{M}$ $+\mathrm{H})^{+}$.

Synthesis of methyl 2-(8-(3-(3-cyanophenyl)ureido) octyl)-6-methoxybenzoate (7h): Using 6 and 3-cyano phenyl isocyanate as starting materials, the title compound 7h was obtained as a pale yellow solid (Yield $=33.5 \%$ ); m.p. $75^{\circ} \mathrm{C}-76^{\circ} \mathrm{C}$; IR (DCM film): 3355, 3083, 3006, 2930, 2855, 2230, 1728, 1664, 1587, 1552, 1470, 1431, 1271, 1111, 1072, 791, $747 \mathrm{~cm}^{-1} ;{ }^{1} \mathrm{H}$ NMR $\left(\mathrm{CDCl}_{3}, 400\right.$ $\mathrm{MHz}): \delta 1.26(\mathrm{bs}, 8 \mathrm{H}), 1.41-1.58(\mathrm{~m}, 4 \mathrm{H}), 2.55(\mathrm{t}, 2 \mathrm{H}, \mathrm{J}$ $=8.0 \mathrm{~Hz}), 3.19-3.24(\mathrm{q}, 2 \mathrm{H}), 3.80(\mathrm{~s}, 3 \mathrm{H}), 3.93(\mathrm{~s}, 3 \mathrm{H})$, $5.2(\mathrm{~s}, 1 \mathrm{H}), 6.78-6.84(\mathrm{~m}, 2 \mathrm{H}), 7.22-7.33(\mathrm{~m}, 3 \mathrm{H})$, 7.57(s, 1H), 7.63 - $7.65(\mathrm{~m}, 1 \mathrm{H}) \mathrm{ppm}$; ESIMS (m/z): 438 $(\mathrm{M}+\mathrm{H})^{+}$.

Synthesis of methyl 2-methoxy-6-(8-(3-(3-(trifluoromethyl)phenyl)ureido)octyl)benzoate (7i): Using 6 and 3-trifluromethyl phenyl isocyanate as starting materials, the title compound $7 \mathbf{i}$ was obtained as a white solid $\left(\right.$ Yield $=42.6 \%$ ); m.p. $99^{\circ} \mathrm{C}-101^{\circ} \mathrm{C}$; IR (DCM film): 3349, 3096, 3005, 2932, 2856, 1730, 1660, 1564, 1442, 1335, 1266, 1120, $1072 \mathrm{~cm}^{-1} ;{ }^{1} \mathrm{H}$ NMR $\left(\mathrm{CDCl}_{3}, 400\right.$ $\mathrm{MHz}): \delta 1.26(\mathrm{bs}, 8 \mathrm{H}), 1.41-1.58(\mathrm{~m}, 4 \mathrm{H}), 2.55(\mathrm{t}, 2 \mathrm{H}, \mathrm{J}$ $=7.6 \mathrm{~Hz}), 3.19-3.24(\mathrm{q}, 2 \mathrm{H}), 3.79(\mathrm{~s}, 3 \mathrm{H}), 3.93(\mathrm{~s}, 3 \mathrm{H})$, $5.12(\mathrm{~s}, 1 \mathrm{H}), 6.77(\mathrm{~d}, 1 \mathrm{H}, \mathrm{J}=8.4 \mathrm{~Hz}), 6.82(\mathrm{~d}, 1 \mathrm{H}, \mathrm{J}=$ $8.0 \mathrm{~Hz}), 7.075(\mathrm{~s}, 1 \mathrm{H}), 7.22-7.36(\mathrm{~m}, 3 \mathrm{H}), 7.55-7.59$ $(\mathrm{m}, 2 \mathrm{H}) \mathrm{ppm}$; ESIMS (m/z): $481(\mathrm{M}+\mathrm{H})^{+}$.

Synthesis of methyl 2-methoxy-6-(8-(3-(3-methoxy phenyl)ureido)octyl)benzoate (7j): Using 6 and 3-methoxy phenyl isocyanate as starting materials, the title compound $\mathbf{7 j}$ was obtained as a off white solid (Yield = $88.4 \%$ ); m.p. $82^{\circ} \mathrm{C}-83^{\circ} \mathrm{C}$; IR (DCM film): 3343, 2935 , 2861, 1726, 1567, 1466, 1266, 1109, 1072, $772 \mathrm{~cm}^{-1} ;{ }^{1} \mathrm{H}$ NMR $\left(\mathrm{CDCl}_{3}, 400 \mathrm{MHz}\right): \delta 1.26(\mathrm{bs}, 8 \mathrm{H}), 1.40-1.58(\mathrm{~m}$, $4 \mathrm{H}), 2.53(\mathrm{t}, 2 \mathrm{H}, \mathrm{J}=8.0 \mathrm{~Hz}), 3.19-3.22(\mathrm{q}, 2 \mathrm{H}), 3.77(\mathrm{~s}$, $3 \mathrm{H}), 3.80(\mathrm{~s}, 3 \mathrm{H}), 3.91(\mathrm{~s}, 3 \mathrm{H}), 5.08(\mathrm{~s}, 1 \mathrm{H}), 6.59-6.60$ $(\mathrm{m}, 1 \mathrm{H}), 6.75-6.82(\mathrm{~m}, 3 \mathrm{H}), 7.05(\mathrm{~s}, 1 \mathrm{H}), 7.12-7.18(\mathrm{~m}$, 1H), 7.25 - $7.30(\mathrm{~m}, 1 \mathrm{H})$ ppm; ESIMS (m/z): $443(\mathrm{M}+$ $\mathrm{H})^{+}$.

Synthesis of methyl 2-(8-(3-(2,2-dimethyl-2,3-dihydro benzofuran-7-yl)ureido)octyl)-6-methoxybenzoate (7k): Using 6 and 7-isocyanato-2, 2-dimethyl-2, 3-dihydro benzofuran as starting materials, the title com- pound 7k was obtained as a light brown color solid (Yield: 
44.4\%); m.p. $96^{\circ} \mathrm{C}-97^{\circ} \mathrm{C}$; IR (DCM film): 3347, 3050, 2929, 2855, 1730, 1658, 1565, 1441, 1374, 1300, 1267, 1111, 1070, 877, 765, $738 \mathrm{~cm}^{-1} ;{ }^{1} \mathrm{H}$ NMR $\left(\mathrm{CDCl}_{3}, 400\right.$ $\mathrm{MHz}): \delta 1.28(\mathrm{bs}, 8 \mathrm{H}), 1.46(\mathrm{~s}, 6 \mathrm{H}), 1.46-1.60(\mathrm{~m}, 4 \mathrm{H})$, $2.53(\mathrm{t}, 2 \mathrm{H}, \mathrm{J}=7.6 \mathrm{~Hz}), 3.03(\mathrm{~s}, 2 \mathrm{H}), 3.21-3.26(\mathrm{q}, 2 \mathrm{H})$, $3.81(\mathrm{~s}, 3 \mathrm{H}), 3.90(\mathrm{~s}, 3 \mathrm{H}), 4.85(\mathrm{~s}, 1 \mathrm{H}), 6.21(\mathrm{~s}, 1 \mathrm{H}), 6.74$ - $6.85(\mathrm{~m}, 4 \mathrm{H}), 7.24-7.28(\mathrm{~m}, 1 \mathrm{H}), 7.54(\mathrm{~d}, 1 \mathrm{H}, \mathrm{J}=8.0$ $\mathrm{Hz}) \mathrm{ppm} ;{ }^{13} \mathrm{C} \mathrm{NMR}\left(100 \mathrm{MHz}, \mathrm{CDCl}_{3}\right), \delta: 26.78,28.20$, 29.09, 29.20, 29.26, 30.01, 30.98, 33.39, 40.30, 43.32, $52.13,55.80,87.56,108.33,119.50,119.69,120.59$, $121.46,122.97,123.36,126.89$, 130.22, 141.24, 148.55, 155.82, 156.20, 168.97 ppm; ESIMS (m/z): $483(\mathrm{M}+$ $\mathrm{H})^{+}$.

Synthesis of methyl 2-methoxy-6-(8-(3-phenyl thioure ido)octyl) benzoate (8a): Using 6 and phenyl thioisocyanate as starting materials, the title compound $\mathbf{8 a}$ was obtained as a light brown liquid (Yield $=50.2 \%$ ); IR (DCM film): 3280, 3054, 3005, 2930, 2854, 1728, 1590, $1536,1502,1465,1304,1267,1110,1072,753 \mathrm{~cm}^{-1} ;{ }^{1} \mathrm{H}$ NMR $\left(\mathrm{CDCl}_{3}, 400 \mathrm{MHz}\right): \delta 1.29$ (bs, 8H), 1.57 (bs, 4H), $2.54(\mathrm{t}, 2 \mathrm{H}, \mathrm{J}=8.0 \mathrm{~Hz}), 3.60-3.64(\mathrm{~m}, 2 \mathrm{H}), 3.82(\mathrm{~s}, 3 \mathrm{H})$, $3.91(\mathrm{~s}, 3 \mathrm{H}), 6.02(\mathrm{~s}, 1 \mathrm{H}), 6.77(\mathrm{~d}, 1 \mathrm{H}, \mathrm{J}=8 \mathrm{~Hz}), 6.82(\mathrm{~d}$, $1 \mathrm{H}, \mathrm{J}=8.0 \mathrm{~Hz}), 7.22$ - $7.34(\mathrm{~m}, 4 \mathrm{H}), 7.43-7.47(\mathrm{~m}, 2 \mathrm{H})$, $7.59(\mathrm{~s}, 1 \mathrm{H}) \mathrm{ppm}$; ESIMS (m/z): $429(\mathrm{M}+\mathrm{H})^{+}$.

Synthesis of methyl 2-(8-(3-(3-chlorophenyl) thioure ido)octyl)-6-methoxybenzoate (8b): Using 6 and 3chloro phenyl thioisocyanate as starting materials, the title compound $\mathbf{8 b}$ was obtained as a off white solid (Yield $=80.3 \%$ ); m.p. $103^{\circ} \mathrm{C}-104^{\circ} \mathrm{C}$; IR (DCM film): 3349, 3083, 3004, 2930, 2855, 1730, 1660, 1592, 1544, 1473, 1429, 1269, 1110, 1073, 773, $741 \mathrm{~cm}^{-1} ;{ }^{1} \mathrm{H}$ NMR $\left(\mathrm{CDCl}_{3}, 400 \mathrm{MHz}\right): \delta 1.25$ (bs, 8H), $1.44-1.60(\mathrm{~m}, 4 \mathrm{H})$, $2.54(\mathrm{t}, 2 \mathrm{H}, \mathrm{J}=7.6 \mathrm{~Hz}), 3.18-3.20(\mathrm{~m}, 2 \mathrm{H}), 3.8(\mathrm{~s}, 3 \mathrm{H})$, $3.92(\mathrm{~s}, 3 \mathrm{H}), 5.2(\mathrm{~s}, 1 \mathrm{H}), 6.77(\mathrm{~d}, 1 \mathrm{H}, \mathrm{J}=8.4 \mathrm{~Hz}), 6.82(\mathrm{~d}$, $1 \mathrm{H}, \mathrm{J}=7.6 \mathrm{~Hz}), 6.95(\mathrm{~d}, 1 \mathrm{H}, \mathrm{J}=8.0 \mathrm{~Hz}), 7.12-7.21(\mathrm{~m}$, 2H), 7.28 - $7.35(\mathrm{~m}, 2 \mathrm{H}) \mathrm{ppm}$; ESIMS (m/z): $463(\mathrm{M}+$ $\mathrm{H})^{+}$.

Synthesis of methyl 2-(8-(3-(3,4-dichlorphenyl)thioureido)octyl)-6-methoxybenzoate (8c): Using 6 and phenyl thioisocyanate as starting materials, the title compound 8c was obtained as a pale brown color liquid (Yield $=98.4 \%$ ); IR (DCM film): 3298, 3055, 3008, 2930, 2855, 1725, 1588, 1533, 1470, 1269, 1115, 1072, 1030, 822, $736 \mathrm{~cm}^{-1}$; ${ }^{1} \mathrm{H}$ NMR $\left(\mathrm{CDCl}_{3}, 400 \mathrm{MHz}\right): \delta$ 1.37 (bs, 8H), 1.58 (s, 4H), 2.55 (t, 2H, J = 7.2 Hz), 3.60 (bs, 2H), $3.81(\mathrm{~s}, 3 \mathrm{H}), 3.91(\mathrm{~s}, 3 \mathrm{H}), 6.06(\mathrm{~s}, 1 \mathrm{H}), 6.77(\mathrm{~d}$, $1 \mathrm{H}, \mathrm{J}=8.0 \mathrm{~Hz}), 6.82(\mathrm{~d}, 1 \mathrm{H}, \mathrm{J}=7.2 \mathrm{~Hz}), 7.13(\mathrm{~d}, 1 \mathrm{H}, \mathrm{J}=$ $8.4 \mathrm{~Hz}), 7.26-7.30(\mathrm{~m}, 1 \mathrm{H}), 7.38(\mathrm{~s}, 1 \mathrm{H}), 7.48(\mathrm{~d}, 1 \mathrm{H}, \mathrm{J}$ $=8.8 \mathrm{~Hz}), 7.67(\mathrm{~s}, 1 \mathrm{H}) \mathrm{ppm} ; \operatorname{ESIMS}(\mathrm{m} / \mathrm{z}): 497(\mathrm{M}+\mathrm{H})^{+}$. 499 (chloro).

Synthesis of methyl 2-(8-(3-(2-fluorophenyl)thioureido)octyl)-6-methoxybenzoate (8d): Using 6 and 2fluorophenyl thioisocyanate as starting materials, the title compound 8d was obtained as a pale brown liquid (Yield =74.3\%); IR (DCM film): 3259, 3050, 3006, 2931, 2855, $1728,1587,1541,1505,1465,1268,1110,1072,956$, $752 \mathrm{~cm}^{-1} ;{ }^{1} \mathrm{H}$ NMR $\left(\mathrm{CDCl}_{3}, 400 \mathrm{MHz}\right): \delta 1.30(\mathrm{bs}, 8 \mathrm{H})$, 1.58 (bs, 4H), 2.54 (t, 2H, J = 8.0 Hz), 3.63 (bs, 2H), $3.82(\mathrm{~s}, 3 \mathrm{H}), 3.92(\mathrm{~s}, 3 \mathrm{H}), 6.04(\mathrm{~s}, 1 \mathrm{H}), 6.77(\mathrm{~d}, 1 \mathrm{H}, \mathrm{J}=$ $8.0 \mathrm{~Hz}), 6.82(\mathrm{~d}, 1 \mathrm{H}, \mathrm{J}=8.0 \mathrm{~Hz}), 7.19-7.41(\mathrm{~m}, 5 \mathrm{H})$ ppm; ESIMS (m/z): $447(\mathrm{M}+\mathrm{H})^{+}$.

Synthesis of methyl 2-(8-(3-(3-cyanophenyl)thioureido)octyl)-6-methoxybenzoate (8e): Using 6 and 3cyanophenyl thioisocyanate as starting materials, the title compound $8 \mathbf{e}$ was obtained as a yellow solid (Yield = $36.6 \%$ ); m.p. $114^{\circ} \mathrm{C}-115^{\circ} \mathrm{C}$; IR (DCM film): 3334,2939 , 2862, 2223, 1725, 1639, 1549, 1466, 1265, 1109, 1071 $\mathrm{cm}^{-1} ;{ }^{1} \mathrm{H}$ NMR $\left(\mathrm{CDCl}_{3}, 400 \mathrm{MHz}\right): \delta 1.33(\mathrm{bs}, 8 \mathrm{H}), 1.58$ (bs, 4H), $1.80-1.82(\mathrm{~m}, 2 \mathrm{H}), 2.53(\mathrm{t}, 2 \mathrm{H}, \mathrm{J}=8.0 \mathrm{~Hz})$, $3.81(\mathrm{~s}, 3 \mathrm{H}), 3.91(\mathrm{~s}, 3 \mathrm{H}), 4.6(\mathrm{~s}, 1 \mathrm{H}), 6.75(\mathrm{~d}, 1 \mathrm{H}, \mathrm{J}=$ $8.4 \mathrm{~Hz}), 6.82(\mathrm{~d}, 1 \mathrm{H}, \mathrm{J}=8.0 \mathrm{~Hz}), 6.95(\mathrm{~d}, 1 \mathrm{H}, \mathrm{J}=8.0 \mathrm{~Hz})$, 7.23 - $7.28(\mathrm{~m}, 3 \mathrm{H}), 7.47$ - $7.51(\mathrm{~m}, 1 \mathrm{H})$ ppm; ESIMS $(\mathrm{m} / \mathrm{z}): 454(\mathrm{M}+\mathrm{H})^{+}$.

Synnthesis of methyl 2-methoxy-6-(8-(3-(3-(trifluoro methyl)phenyl)thioureido)octyl) benzoate (8f): Using 6 and 3-trifluromethylphenyl thioisocyanate as star- ting materials, the title compound $\mathbf{8 f}$ was obtained as a light yellow liquid (Yield $=46.7 \%$ ); IR (DCM film): 3325 , 3070, 3013, 2931, 2856, 1725, 1666, 1582, 1459, 1331, 1269, 1119, 1073, $894 \mathrm{~cm}^{-1}$; ${ }^{1} \mathrm{H}$ NMR $\left(\mathrm{CDCl}_{3}, 400\right.$ $\mathrm{MHz}): \delta 1.288(\mathrm{bs}, 8 \mathrm{H}), 1.58(\mathrm{bs}, 4 \mathrm{H}), 2.53(\mathrm{t}, 2 \mathrm{H}, \mathrm{J}=$ $8.0 \mathrm{~Hz}), 3.61(\mathrm{bs}, 2 \mathrm{H}), 3.79(\mathrm{~s}, 3 \mathrm{H}), 3.90(\mathrm{~s}, 3 \mathrm{H}), 6.06(\mathrm{~s}$, $1 \mathrm{H}), 6.75(\mathrm{~d}, 1 \mathrm{H}, \mathrm{J}=8.0 \mathrm{~Hz}), 6.81(\mathrm{~d}, 1 \mathrm{H}, \mathrm{J}=8.0 \mathrm{~Hz})$, 7.25 - $7.29(\mathrm{~m}, 1 \mathrm{H}), 7.46-7.55(\mathrm{~m}, 4 \mathrm{H}), 7.74(\mathrm{~s}, 1 \mathrm{H})$ ppm; ESIMS (m/z): 495(M - H) ${ }^{+}$.

Antibacterial Bioassay [27]: Urea and thiourea derivatives of Anacardic acid (7a-7k, 8a-8f) were dissolved in dimethyl sulphoxide at $250 \mu \mathrm{g} / \mathrm{mL}$ concentration. The composition of nutrient agar medium was Bactotryptone $(10 \mathrm{~g})$, yeast extract $(5 \mathrm{~g}), \mathrm{NaCl}(10 \mathrm{~g})$, final $\mathrm{pH}$ 7.4. After $18 \mathrm{~h}$ the exponentially growing cultures of the six bacteria in nutrient broth at $37^{\circ} \mathrm{C}$ were diluted in sterile broth. From each of these diluted cultures, $1 \mathrm{~mL}$ was added to $100 \mathrm{~mL}$ sterilized and cooled nutrient agar media to give a final bacterial count of $1 \times 10^{6} \mathrm{cell} / \mathrm{ml}$. The plates were set at room temperature and later dried at $37^{\circ} \mathrm{C}$ for $20 \mathrm{~h}$. Paper discs $(6 \mathrm{~mm}$, punched from Whatmann no. 41 paper) were ultraviolet sterilized and used for the assays. Discs were soaked in different concentration of the test solution and placed on the inoculated agar media at regular intervals of $6-7 \mathrm{~cm}$, care was taken to ensure that excess solution was not on the discs. All the samples were taken in triplicates. The plates were incubated at $37^{\circ} \mathrm{C}$ in an inverted fashion. Activity was determined by zones showing complete inhibition (mm). Growth inhibition was calculated with reference to positive 
control.

\section{Acknowledgements}

We thank GVK Biosciences Private Limited for the financial support and encouragement. We also thankful to the analytical department for their analytical data and to Dr. Balaram Patro for his helpful suggestions.

\section{References}

[1] J. D. Mitchell and S. A. Mori, "The Cashew and Its Relatives (Anacardium:Anacardiaceae)," New York Botanical Garden, New York, 1987.

[2] D. V. Johnson, "O Caju do Nordeste do Brasil-Um Estudo Geográfico,” ETENE/BNB, 1974.

[3] J. S. Aggarwal, "Cashewnut Shell Liquid: Part I-Chemistry, Chemicals and Other Useful Products from It," Journal of the Colour Society, Vol. 14, No. 3, 1975, pp. 1-9.

[4] M. S. Ramaiah, "Progress of Research in Cashew Industry," Fette, Seifen, Anstrichmittel, Vol. 78, 1976, pp. 472477. doi:10.1002/lipi.19760781204

[5] O. Attanasi, F. Serra-Zanetti, F. Perdomi and A. Scagliarini, La Chimica \& L'Industria, Vol. 61, 1979, pp. 718-725.

[6] J. H. P. Tyman, Chemistry \& Industry, Vol. 2, 1980, pp. 59.

[7] J. H. P. Tyman, "Non-Isoprenoid Long Chain Phenols," Chemical Society Reviews, Vol. 8, No. 4, 1979, pp. 499537. doi:10.1039/cs9790800499

[8] I. Kubo, H. Muroi, M. Himejima and Y. Yamigiwa, "Structure-Antibacterial Activity Relationships of Anacardic Acids," Journal of Agricultural and Food Chemistry, Vol. 41, No. 6, 1993, pp. 1016-1019. doi:10.1021/jf00030a036

[9] J. L. Gillerman, N. J. Walsh, N. K. Werner and H. Schlenk, "Antimicrobial Effects of Anacardic Acids," Canadian Journal of Microbiology, Vol. 15, 1969, pp. 1219-1213.

[10] M. Toyomizu, K. Okamoto, T. Ishibashi, Z. Chen and T. Nakatsu, "Uncoupling Effect of Anacardic Acids from Cashew Nut Shell Oil on Oxidative Phosphorylation of Rat Liver Mitochondria," Life Sciences, Vol. 66, No. 3, 2000, pp. 229-234. doi:10.1016/S0024-3205(99)00585-8

[11] B. Prithiviraj, M. Manickam, V. P. Singh and A. B. Ray, "Antifungal Activity of Anacardic Acid, a Naturally Occurring Derivative of Salicylic Acid," Canadian Journal of Botany, Vol. 75, No. 1, 1997, pp. 207-211. doi:10.1139/b97-021

[12] H. Muroi and I. Kubo, "Antibacterial Activity of Anacardic Acid and Totarol, alone and in Combination with Methicillin, against Methicillinresistant Staphylococcus Aureus," Journal of Applied Microbiology, Vol. 80, No. 4, 1996, pp. 387-394.
doi:10.1111/j.1365-2672.1996.tb03233.X

[13] J. Kubo, J. R. Lee and I. Kubo, “Anti-Helicobacter Pylori Agents from the Cashew Apple," Journal of Agricultural and Food Chemistry, Vol. 47, No. 2, 1999, pp. 533-537. doi:10.1021/jf9808980

[14] I. Kubo, H. Muroi and M. Himejima, "Structure-Anti Bacterial Activity Relationships of Anacardic Acids," Journal of Agricultural and Food Chemistry, Vol. 41, 1993, p. 1016.

[15] A. S. Gulati and B. C. Subba Rao, Indian Journal of Chemistry, Vol. 2, 1964, p. 337.

[16] M. A. EIShol, P. D. Adawadkar, D. A. Benign, E. S. Watson and T. L. Little Jr., "6-(Alkylamino)-9-benzyl9H-purines. A New Class of Anticonvulsant Agents," Journal of Medicinal Chemistry, Vol. 29, 1986, pp. 606612.

[17] R. Paramashivappa, P. P. Kumar, P. V. S. Rao and S. A. Rao, "Design, Synthesis and Biological Evaluation of Benzimidazole/Benzothiazole and Benzoxazole Derivatives as Cyclooxygenase Inhibitors," Bioorganic \& $\mathrm{Me}$ dicinal Chemistry Letters, Vol. 13, No. 4, 2003, pp. 657-660. doi:10.1016/S0960-894X(02)01006-5

[18] M. A. Elsholy, P. D. Adawadkar, D. A. Beniggni, E. S. Watson and T. L. Little Jr., "Analogs of Poison Ivy Urushiol. Synthesis and Biological Activity of Disubstituted N-Alkylbenzenes," Journal of Medicinal Chemistry, Vol. 29, No. 5, 1986, pp. 606-611. doi: $10.1021 / \mathrm{jm} 00155 \mathrm{a} 003$

[19] L. S. Kiong and J. H. P. Tyman, "Long-Chain Phenols. Part 18. Conversion of Anacardic Acid into Urushiol," Journal of the Chemical Society, Perkin Transactions 1, 1981, pp. 1942-1952. doi:10.1039/p19810001942

[20] A. S. Gulati and B. C. S. Subba Rao, Indian Journal of Chemistry, Vol. 2, 1964, p. 339.

[21] S. V. Shoba, C. S. Ramadoss and B. Ravindranath, "Inhibition of Soybean Lipoxygenase-1 by Anacardic Acids, Cardols, and Cardanols," Journal of Natural Products, Vol. 57, No. 12, 1994, pp.1755-1757. doi: $10.1021 / \mathrm{np} 50114 \mathrm{a} 025$

[22] T. J. Ha and I. Kubo, "Lipoxygenase Inhibitory Activity of Anacardic Acids," Journal of Agricultural and Food Chemistry, Vol. 53, No. 11, 2005, pp. 4350-4354. doi:10.1021/jf048184e

[23] I. Kubo, M. Ochi, P. C. Viera and S. Komatsu, Journal of Agricultural and Food Chemistry, Vol. 41, 1993, pp. 1012-1015.

[24] M. J. Rybak and R. L. Akins, "Emergence of Methicillin-Resistant Staphylococcus Aureus with Intermediate Glycopeptide Resistance: Clinical Significance and Treatment Options," Drugs, Vol. 61, No. 1, 2001, pp. 1-7. doi:10.2165/00003495-200161010-00001

[25] M. Lipsitch, "The Rise and Fall of Antimicrobial Resistance," Trends in Microbiology, Vol. 9, No. 9, 2001, pp. 438-444. doi:10.1016/S0966-842X(01)02130-8

[26] B. A. Cunha, Drugs Today, Vol. 34, 1998, pp. 691-698.

[27] A. Marchese, G. C. Schito and E. A. Debbia, Journal of 
Chemotherapy, Vol. 12, 2000, pp. 459-462.

[28] Y. Cetinkaya, P. Falk and C. G. Mayhall, "VancomycinResistant Enterococci," Clinical Microbiology Reviews, Vol. 13, No. 4, 2000, pp. 686-707. doi:10.1128/CMR.13.4.686-707.2000

[29] G. H. Cassell and J. Mekalanos, "Development of Antimicrobial Agents in the Era of New and Reemerging Infectious Diseases and Increasing Antibiotic Resistance," Journal of the American Medical Association, Vol. 285, No. 5, 2001, pp. 601-605. doi:10.1001/jama.285.5.601

[30] D. T. W. Chu, J. J. Plattner and L. Katz, "New Directions in Antibacterial Research," Journal of Medicinal Chemistry, Vol. 39, No. 20, 1996, pp. 3853-3874. doi:10.1021/jm960294s

[31] V. Chandregowda, A. Kush and G. C. Reddy, "Synthesis of Benzamide Derivatives of Anacardic Acid and Their Cytotoxic Activity," European Journal of Medicinal Chemistry, Vol. 44, No. 6, 2009, pp. 2711-2719. doi:10.1016/j.ejmech.2009.01.033

[32] R. Paramashivappa, P. Phanikumar, P. V. Subba Rao and A. Srinivasa Rao, "Synthesis of Sildenafil Analogues from Anacardic Acid and Their Phosphodiesterase-5 Inhibition," Journal of Agricultural and Food Chemistry, Vol. 50, No. 26, 2002, pp. 7709-7713. doi:10.1021/if0258050

[33] P. Phanikumar, S. C. Stotz, R. Paramashivappa, M. Beedle, G. W. Zamponi and A. Srinivasa Rao, "Synthesis and Evaluation of a New Class of Nifedipine Analogs with T-Type Calcium Channel Blocking Activity," Molecular Pharmacology, Vol. 61, No. 3, 2002, pp. 649- 658. doi:10.1124/mol.61.3.649

[34] B. Narayana Swamy, T. K. Suma, G. Venkateswara Rao and G. Chandrasekara Reddy, European Journal of Medicinal Chemistry, Vol. 42, 2007, pp. 422424.

[35] K. Mantelingu, A. H. Kishore, K. Balasubramanyam, G. V. Kumar, M. Altaf, S. N. Swamy, R. Selvi, C. Das, C. Narayana, K. S. Rangappa and T. K. Kundu, "Activation of p300 Histone Acetyltransferase by Small Molecules Altering Enzyme Structure: Probed by Surface-Enhanced Raman Spectroscopy," Journal of Physical Chemistry B, Vol. 111, No. 17, 2007, pp.4527-4534. doi:10.1021/jp067655s

[36] A. Hari Kishore, B. M. Vedamurth, K. Mantelingu, S. Agrawal, B. A. Ashok Redd, S. Roy, K. S. Rangappa and T. K. Kundu, "Specific Small-Molecule Activator of Aurora Kinase A Induces Autophosphorylation in a CellFree System," Journal of Medicinal Chemistry, Vol. 51, No. 4, 2008, pp. 792-797. doi:10.1021/jm700954w

[37] B. Sung, M. K. Pande, K. S. Ahn, T. Yi, M. M. Chaturved, M. Liu and B. B. Aggarwal, "Anacardic Acid
(6-Nonadecyl Salicylic Acid), an Inhibitor of Histone Acetyltransferase, Suppresses Expression of Nuclear Factor- $\kappa B-R e g u l a t e d$ Gene Products Involved in Cell Survival, Proliferation, Invasion, and Inflammation through Inhibition of the Inhibitory Subunit of Nuclear Factor- $\kappa \mathrm{B} \alpha$ Kinase, Leading to Potentiation of Apoptosis," Blood, Vol. 111, No. 10, 2008, pp. 4880-4891.

doi:10.1182/blood-2007-10-117994

[38] L. Cui, J. Miao, T. Furuya, Q. Fan, X. Li, P. K. Rathod, X. Z. Su and L. Cui, "Histone Acetyltransferase Inhibitor Anacardic Acid Causes Changes in Global Gene Expression during in Vitro Plasmodium Falciparum Development," Eukaryotic Cell, Vol. 7, No. 7, 2008, pp. 12001210. doi:10.1128/EC.00063-08

[39] G. Sbardella, S. Castellano, C. Vicidomini, D. Rotili, A. Nebbioso, M. Miceli, L. Altucci and A. Mai, "Identification of Long Chain Alkylidenemalonates as Novel Small Molecule Modulators of Histone Acetyltransferases," Bioorganic \& Medicinal Chemistry Letters, Vol. 18, No. 9, 2008, pp. 2788-2792. doi:10.1016/j.bmcl.2008.04.017

[40] M. L. Dos Santos and G. C. de Magalhães, Química Nova, Vol. 16, 1993, p. 534.

[41] M. G. Carvalho, R. Braz-Filho, M. L. Dos Santos and G. C. de Magalhães, Journal of the Brazilian Chemical Society, Vol. 4, 1993, pp. 158-164.

[42] M. L. Dos Santos and G. C. de Magalhães, "Utilisation of Cashew Nut Shell Liquid from Anacardium Occidentale as Starting Material for Organic Synthesis: A Novel Route to Lasiodiplodin from Cardols," Journal of the Brazilian Chemical Society, Vol. 10, No. 1, 1999, pp. 13-18. doi:10.1590/S0103-50531999000100003

[43] Lúcio P. L. Logrado, Dâmaris Silveira, Luiz A. S. Romeiro, Manoel O. de Moraes, Bruno C. Cavalcanti, Letícia V. Costa-Lotufo, Cláudia do Ó Pessoa and Maria Lucilia dos Santos, Journal of the Brazilian Chemical Society, Vol. 16, 2005, pp. 1217-1225.

[44] L. S. Kiong and J. H. P. Tyman, "Long-Chain Phenols. Part 18. Conversion of Anacardic Acid into Urushiol," Journal of the Chemical Society, Perkin Transactions 1, Vol. 1, 1981, p. 1942. doi:10.1039/p19810001942

[45] R. Paramashivappa, P. Phanikumar, P. J. Vithayathil and A. Srinivasa Rao, "Novel Method for Isolation of Major Phenolic Constituents from Cashew (Anacardium occidentale L.) Nut Shell Liquid," Journal of Agricultural and Food Chemistry, Vol. 49, No. 5, 2001, pp. 2548-2551. doi:10.1021/jf001222j

[46] A. Rahman, M. L. Choudhary and W. J. Thomsen, "Bioassay Techniques for Drug Development," Harwood Academic Publishers, The Netherlands, 2001. 